\title{
Compendium of Single Event Effects Test Results for Commercial Off-The-Shelf and Standard Electronics for Low Earth Orbit and Deep Space Applications
}

\author{
Brandon D. Reddell, Charles R. Bailey, Patrick M. O’Neill, Kyson V. Nguyen, Razvan Gaza, Chirag \\ Patel, Jaime Cooper, Theodore Kalb, Elden Beach and Larry Mason
}

\begin{abstract}
We present the results of Single Event Effects (SEE) testing with high energy protons and with low and high energy heavy ions for electrical components considered for Low Earth Orbit (LEO) and for deep space applications.
\end{abstract}

\section{INTRODUCTION}

A NASA continues to operate the International Space Station (ISS) in Low Earth Orbit (LEO), it is facilitating the commercialization of LEO by working with companies through the Commercial Crew Program. Relevant to the design and operation of hardware in this environment, there is a need to select electronic components that are known to function for various mission durations. The environments here are relatively benign with occasional passes through the South Atlantic Anomaly region of the trapped proton Van Allen belt. Certification has primarily been carried out through high energy proton testing, which has been successfully used to test for Single Event Effects (SEE) in LEO for over two decades in the Space Shuttle and ISS programs [1]-[2]. It is anticipated that high energy protons will continue to be used by companies intending to fly short duration programs in LEO.

The new focus of the human space exploration program at NASA is focused on destinations in cis-lunar space and eventually to Mars with the Orion Multi-purpose Crew Vehicle being developed by the Lockheed Martin Corporation. Additionally, smaller-scale projects such as small satellites, robotic rovers, and various science payloads will be exposed to similar environments. For all of these missions, the hardware will be exposed to Galactic Cosmic Radiation (GCR) and possibly Solar Particle Events (SPE). SPEs are primarily proton events, but contain concentrations of heavy ions, whereas GCR are heavy ions ranging from hydrogen through iron spanning many orders of magnitude in energy. For these missions, program performance and reliability requirements necessitate the need for heavy ion certification. To date, this

Manuscript received July 10, 2017. (Write the date on which you submitted your paper for review.) This work was supported in part by the U.S. Department of Commerce under Grant No. BS123456 (sponsor acknowledgment goes here).

B. D. Reddell, C. R. Bailey, P. M. O’Neill, and K. V. Nguyen are with the NASA Johnson Space Center, Electronic Design and Manufacturing Branch, 2101 NASA Parkway, Houston, Texas 77058. has been carried out by traditional (low energy) heavy ion testing as well as using the Variable Depth Bragg Peak (VDBP) method for part characterization and for destructive screening.

NASA has primarily conducted proton testing at the Indiana Cyclotron Facility until closure in December 2014, and afterwards, at the Francis Burr Proton Facility (FBPTC) in Boston, Ma. For heavy ions, NASA continues to use Lawrence Berkeley National Laboratory (LBNL) and the Texas A\&M Cyclotron Facility (TAMU) for low energy testing. For high energy testing, NASA has been using the techniques developed by the NASA Johnson Space Center to use the high energy beams at Brookhaven National Laboratory at the NASA Space Radiation Laboratory [3]-[5].

This paper summarizes the test results through the year 2016 in the above mentioned programs and provides generic information to allow the user to evaluate radiation performance for various radiation environments.

\section{TEST PROTOCOL}

\section{A. Proton testing}

NASA uses $200 \mathrm{MeV}$ protons to test for destructive and nondestructive errors for hardware intended for LEO, i.e. for the International Space Station (ISS) [6]-[7]. This test exposes most known failure modes that have a Mean Time Before Failure $(\mathrm{MTBF})<=10$ years in the LEO environment. Proton testing replicates approximately 6-10 years of the heavy ion linear energy transfer (LET) environment up to an LET of approximately $10-14 \mathrm{MeV}-\mathrm{cm}^{2} / \mathrm{mg}$ in silicon. The proton beam typically loses less than $10 \%$ of its energy while passing through the electronic parts. Secondary recoils are typically produced though the inelastic collisions of individual protons with the nuclei in the device, which is primarily silicon, but may contain higher charge elements such as tungsten.

(email: brandon.d.reddell@nasa.gov, charles.r.bailey@nasa.gov, patrick.m.oneill@nasa.gov, and Kyson.v.nguyen@nasa.gov)

R. Gaza, C. Patel, J. Cooper, T. Kalb, E. Beach and L. Mason are with Lockheed Martin Space Systems Company, Houston, Texas, 77058.

(email: razvan.gaza@lmco.com, chirag.patel@lmco.com, jaime.cooper@1mco.com, theodore.kalb@1mco.com, elden.beach@1mco.com, and larry.mason@1mco.com. 
The typical test exposes the device under test to a fluence of $\geq 1 \mathrm{E}+10$ protons $/ \mathrm{cm} 2$ which accomplishes two goals. The first is to find single event effects caused by heavy ions up to LET of $\sim 10 \mathrm{MeV}-\mathrm{cm}^{2} / \mathrm{mg}$. Secondly, the test produces a total ionizing dose (TID) of at least $600 \mathrm{rads}(\mathrm{Si})$, which corresponds to about 10 years of total ionizing dose exposure in LEO.

This NASA method does not fully characterize the part, but it intends to screen for hard failures and provides very conservative estimates up to a 10 year MTBF in LEO [6]-[8]. This test is typically performed at the board or box level which provides a means to reduce the cost of testing, especially with modern Commercial Off-The-Shelf (COTS) units. The test can be used for down-selection for both LEO and deep space applications as well as provide conservative SEE and TID results.

\section{B. Traditional Heavy Ion Testing}

NASA uses traditional methods to perform heavy ion testing and requires each part be characterized to high LET (depending on mission) or failure. Traditional methods require delidding of the parts for single piece part testing and characterization. Often times, components with specific application voltages representative of flight like conditions are tested to understand transient radiation induced responses to these devices or test for the effectiveness of mitigation strategies. Analyses of the SEE signatures at the system level are required to determine the system effects and what mitigations are necessary. Testing complex parts and applying those results to complex systems is a difficult task. The radiation analysis typically involves circuit analysis to evaluate the system level effects while cataloging the effects of each part in the system.

\section{High Energy Heavy Ion Testing}

Increasingly, the human rated missions are incorporating complex parts that are too difficult (or costly) to delid or have sensitive volume depths unreachable by low energy heavy ion beams. This problem has been encountered on the Orion Multipurpose Crewed Vehicle program [9]. Additionally, designs include more Commercial Off-The-Shelf (COTS) units to support crew activities for which there are no rad-hard versions available. In these cases, the traditional test facilities at TAMU and LBNL cannot provide beams with enough energy to penetrate these devices. Furthermore, NASA JSC is seeing a trend towards screening flight boards to certain LET levels for destructive effects while also using the high energy beams to evaluate flight circuits for evaluation of system level soft errors. For individual parts characterization, we implore the Variable Depth Bragg Peak (VDBP) method [3]-[5] and for screening, a modified VDBP method which uses the various degrader steps to ensure all locations in the board are exposed to a certain LET level desired by the program. All VDBP testing reported in this report was accomplished using the ion beams listed in Table 1. The NSRL staff have published an overview of the NSRL facility with more details on beam ion selection and other beam characteristics [10].

Table1

LET (Si) for Ion Beams Used For VDBP Testing

\begin{tabular}{c|cccc|} 
& $\begin{array}{c}\text { Max } \\
\text { Energy } \\
(\mathbf{M e V} / \mathbf{n})\end{array}$ & $\begin{array}{c}\text { LET at Max E } \\
(\mathbf{M e V}- \\
\mathbf{c m} / \mathbf{m g})\end{array}$ & $\begin{array}{c}\text { Peak LET } \\
(\mathbf{M e V}- \\
\mathbf{c m} 2 / \mathbf{m g}) * *\end{array}$ & $\begin{array}{c}\text { Range } \\
\text { in Si } \\
(\mathbf{m m})\end{array}$ \\
\hline $\mathrm{H}^{1}$ & 2500 & 0.00171 & 0.51 & 5470 \\
$\mathrm{C}^{12}$ & 1500 & 0.06227 & 5.2 & 972 \\
$\mathrm{Fe}^{56}$ & 1470 & 1.171 & 29.3 & 235 \\
$\mathrm{Kr}^{84}$ & 383 & 3.28 & 41 & 26.5 \\
$\mathrm{Xe}^{132}$ & 350 & 7.7 & 69.2 & 16.3 \\
$\mathrm{Ta}^{181}$ & 342 & 14.8 & 87.5 & 12.1 \\
$\mathrm{Au}^{197}$ & 165 & 24.7 & 94.2 & 3.7
\end{tabular}

* This table was reproduced from the official listing at https://www.bnl.gov/nsrl/userguide/beam-ion-species-andenergies.php

**This represents the Peak LET of a single particle. The average LET of will be lower because of the energy spread of the beam, and this depends on energy.

For VDBP destructive screening, a series of degrader steps are used to slide the Bragg curve through the whole device. Where the Bragg curves intersect will define a minimum LET exposure at all locations in that device and this value is a function of the degrader step size used. Figure 1 shows the 165 $\mathrm{MeV} / \mathrm{n}$ Au beam with $0.3 \mathrm{~mm}$ of degrader will expose the whole part to an LET of $69 \mathrm{MeV}-\mathrm{cm} 2 / \mathrm{mg}$ or higher.

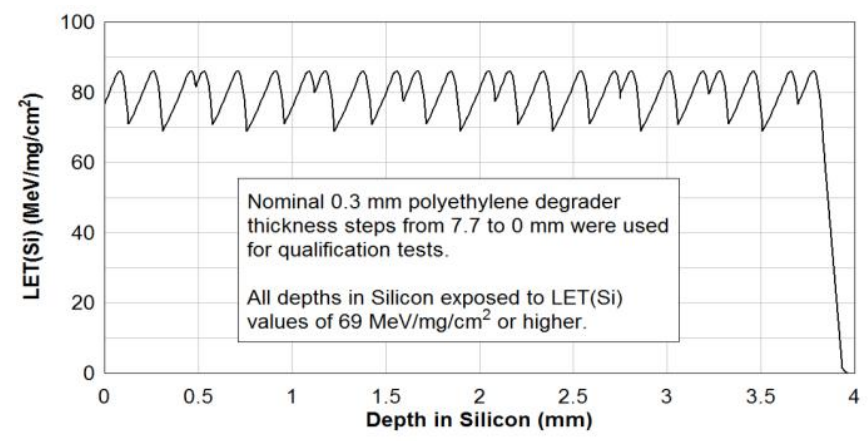

Fig. 1. Example of destructive screening with $165 \mathrm{MeV} / \mathrm{n}$ Au ion with $0.3 \mathrm{~mm}$ steps of polyethylene degrader. A part with $3.5 \mathrm{~mm}$ of $\mathrm{Si}$ (equivalent) will be exposed to a minimum of $\mathrm{LET}=69 \mathrm{MeV}-\mathrm{cm}^{2} / \mathrm{mg}$.

\section{TEST RESULTS OVERVIEW}

Table 2 is an excerpt from the testing results table that includes over 100 parts. For each part, the table gives the report number, the part number, the Lot Date Code (LDC), part type, manufacturer, where it was tested and to what LET and characterization data when determined, i.e. a set of Weibull parameters for heavy ion data and Bendel parameter for proton test data, along with important notes. The LDC's are provided for the tested parts because part manufacturing variations are known to affect radiation susceptibility and this information is 


\begin{tabular}{|c|c|c|c|c|c|}
\hline \multicolumn{6}{|c|}{ Table 2: Summary of SEE Test Results } \\
\hline Part \# & $\underline{\text { LDC }}$ & Part type & Manufacturer & Facility & Weibull Parameters \\
\hline$\overline{\text { OLF300 }}$ & $\overline{504}$ & Opto & Iso Link & $\overline{\text { TAMU }}$ & $\sigma_{0}=1.4 \mathrm{E}-4, \mathrm{Max} S E T=4 \overline{\mathrm{mV}, \text { Max LET }=80.2}$ \\
\hline RH1499 & $1002 \mathrm{~A}$ & Quad opamp & $\mathrm{AD}$ & TAMU & $\begin{array}{l}\mathrm{L}_{0}=15, \sigma_{0}=8.5 \mathrm{E}-3, \mathrm{~W}=19, \mathrm{~S}=1.4, \text { SET pos }=2 \mathrm{us},, 2 \mathrm{~V}, \mathrm{SET} \text { neg }=0, \text { Max } \\
\mathrm{LET}=80.2\end{array}$ \\
\hline REF02 & $1025 \mathrm{~A}$ & Volt Reg & $\mathrm{AD}$ & LBNL & No SET above $6 \mathrm{~V}, \mathrm{~V} \sup =15$, Vout $=5 \mathrm{~V}, \mathrm{Max}$ LET $=75.7$ \\
\hline RH1016MW & $1011 \mathrm{~A}$ & High Speed Comp & LT & LBNL & $\mathrm{L}_{0}=12.5, \sigma_{0}=6 \mathrm{E}-5, \mathrm{~W}=50, \mathrm{~S}=2.5, \mathrm{SET}=+/-0.5 \mathrm{~V}, \mathrm{Max} \mathrm{LET}=75.7$ \\
\hline SNV54AHC244W & 0726A & Octal buffer & TI & LBNL & $\begin{array}{l}\text { No SET between } 1.3 \mathrm{~V} \text { and } 0.8 \mathrm{~V} \text {. Operating at } 3.3 \mathrm{~V} \text {, } \\
\text { No DSEE, Max LET }=75.7\end{array}$ \\
\hline HYSE-117RH-Q & $\begin{array}{l}\text { Proto - } \\
\text { same as } \\
\text { flight }\end{array}$ & Adj Pos volt reg & Intersil & LBNL & $\mathrm{L}_{0}=7, \sigma_{0}=1.2 \mathrm{E}-4, \mathrm{~W}=40, \mathrm{~S}=3$ \\
\hline OLI249 & 114814 & Opto & Isolink & LBNL & $\begin{array}{l}\mathrm{L}_{0}=25, \sigma_{0}=6.0 \mathrm{E}-3, \mathrm{~W}=50, \mathrm{~S}=4, \mathrm{SET}>=2.2 \mathrm{~V}, \mathrm{~V} \text { sup and } \mathrm{Vout}=3.3 \mathrm{~V}, \\
60 \mathrm{us} \text { duration maximum, Max LET }=75.7\end{array}$ \\
\hline RH1014MW & $1101 \mathrm{~A}$ & Quad op amp & LT & TAMU & $\begin{array}{l}\mathrm{L}_{0}=8.6, \sigma_{0}=8.4 \mathrm{E}-4, \mathrm{~W}=32, \mathrm{~S}=3 \text {, Max POS SET }=8 \mathrm{~V} / 22 \mathrm{us} \\
\mathrm{L}_{0}=1.8, \sigma_{0}=7.0 \mathrm{E}-3, \mathrm{~W}=26, \mathrm{~S}=1.7 \text {, Max NEG SET }=-9 \mathrm{~V} / 60 \mathrm{us} \\
\text { Max LET }=87.1\end{array}$ \\
\hline LMC6484 & 1043 & Quad op amp & $\begin{array}{c}\text { National } \\
\text { Semiconductor }\end{array}$ & TAMU & No SEL, SET < 10us, Max LET=87.1 \\
\hline HS-4423BRH & $\mathrm{X} 1006 \mathrm{~A}$ & $\begin{array}{l}\text { Dual Inv MOSFET } \\
\text { Driver } \\
\end{array}$ & Intersil & TAMU & $\begin{array}{l}\mathrm{L}_{0}=19, \sigma_{0}=2.94 \mathrm{E}-05, \mathrm{~W}=16.03, \mathrm{~S}=1.926 \\
80 \mathrm{nS} \text { transients, Max LET }=85.4\end{array}$ \\
\hline 54LVC08A & $1217 \mathrm{~A}$ & $\begin{array}{l}\text { Quadruple 2-input } \\
\text { AND Gate }\end{array}$ & TI & IUCF & $200 \mathrm{MeV}, 1 \mathrm{E} 10 / \mathrm{cm}^{2}$ \\
\hline SG7805AT & 7C1143P & 5V Voltage Regulator & Microsemi & IUCF & $200 \mathrm{MeV}, 5 \mathrm{E} 10 / \mathrm{cm}^{2}$ \\
\hline IS705RH & $1113 \mathrm{AC}$ & $\begin{array}{l}\text { Power-up/down } \\
\text { Microprocessor Reset } \\
\text { Circuit }\end{array}$ & Intersil & NSRL & $\begin{array}{l}\text { SET: } \sigma_{0}=7.47 \mathrm{E}-5, \mathrm{~L}_{0}=22, \mathrm{~W}=26.85, \mathrm{~s}=3.09, \text { No } \mathrm{SEL} \text { to } \mathrm{LET}=60 \text { with } \\
1 \mathrm{E} 6 / \mathrm{cm}^{2}\end{array}$ \\
\hline IRHM7360SE & 542 & $\begin{array}{l}\text { 400V N-Channel } \\
\text { MOSFET }\end{array}$ & $\begin{array}{l}\text { International } \\
\text { Rectifier }\end{array}$ & TAMU & $176 \mathrm{~V}$ at $40 \mathrm{LET}$ pass voltage (9KOhm load) \\
\hline IRHF7330SE & 918 & $\begin{array}{l}\text { 400V N-Channel } \\
\text { MOSFET }\end{array}$ & $\begin{array}{l}\text { International } \\
\text { Rectifier }\end{array}$ & TAMU & Pass at $182 \mathrm{~V}$ at $\mathrm{LET}=40$, (shorted output) \\
\hline SCF128XFTG64C & 1201 & 128M NV Flash ROM & Xilinx & IUCF & No switched bits \\
\hline IRHY57230CMSE & 1110 & $\begin{array}{l}\text { 200V N-Channel } \\
\text { MOSFET }\end{array}$ & $\begin{array}{l}\text { International } \\
\text { Rectifier }\end{array}$ & TAMU & Pass at $182 \mathrm{~V}$ at LET $=85.4$, (shorted output) \\
\hline 66099 & 1046 & Optocoupler & Micropac & IUCF & Bendel FIT A=15.48 (single point) \\
\hline 66252 & 1219 & Optocoupler & Micropac & IUCF & No SET observed, $15 \mathrm{~V}$ and $3.3 \mathrm{~V}$ bias \\
\hline UC1845 & 0910A & PWM & TI & LBNL & $\begin{array}{l}\mathrm{L}_{0}=3, \sigma_{0}=2.0 \mathrm{E}-3, \mathrm{~W}=28, \mathrm{~S}=1.3, \mathrm{SET} \text { at }+/-50 \% \text { duty cycle, Max } \\
\mathrm{LET}=75.66\end{array}$ \\
\hline SNV54AC14W-SP & $1131 \mathrm{~A}$ & $\begin{array}{l}\text { Hex inverter, Schmidt } \\
\text { tr. }\end{array}$ & IR & TAMU & SET Lo >39.9, Low-high SET from ground to rail (3.3) at LET=85.4, \\
\hline AD589 & 231 & Volt Reference & $\mathrm{AD}$ & LBNL & $\begin{array}{l}\text { SET1: Short positive (less than } 50 \mathrm{~ns} \text { ) followed by } 8 \mathrm{uS} \text { negative } 400 \mathrm{mV} \\
\text { Weibull: } \mathrm{L}_{0}=2, \sigma_{0}=4.0 \mathrm{E}-4, \mathrm{~W}=2, \mathrm{~S}=1.1 \\
\text { SET 2: } 1 \mathrm{us}, 500 \mathrm{mV} \text {. } \\
\text { Weibull: } \mathrm{L}_{0}=24, \sigma_{0}=2.0 \mathrm{E}-4, \mathrm{~W}=20, \mathrm{~S}=1 \text {, both tested at } \mathrm{LET}=75 \text {, No } \\
\text { DSEE }\end{array}$ \\
\hline OP27 & 145 & Op-amp & $\mathrm{AD}$ & LBNL & $\begin{array}{l}\text { SET1: } 1 \mathrm{~V}, 150 \mathrm{uS} \\
\text { Weibull: } \mathrm{L}_{0}=3.4, \sigma_{0}=8.0 \mathrm{E}-4, \mathrm{~W}=15, \mathrm{~S}=2\end{array}$ \\
\hline
\end{tabular}




\begin{tabular}{|c|c|c|c|c|c|}
\hline & & & & & $\begin{array}{l}\text { SET2: }-500 \mathrm{mV}, 150 \mathrm{uS} \text {. } \\
\text { Weibull: } \mathrm{L}_{0}=25, \sigma_{0}=1.0 \mathrm{E}-4, \mathrm{~W}=20, \mathrm{~S}=4,12 \mathrm{~V} \text { bias, output }=2 \mathrm{~V} \text {, No } \\
\text { DSEE, Max LET }=75.6\end{array}$ \\
\hline $66260-300$ & 1104 & Optocoupler & Micropac & LBNL & $\begin{array}{l}\text { Vcc }=3.3 \mathrm{~V} \\
\text { SET: } 1 \mathrm{~V}, 40 \mathrm{us} \\
\text { Weibull: } \mathrm{L}_{0}=3.2, \sigma_{0}=2.0 \mathrm{E}-3, \mathrm{~W}=20, \mathrm{~S}=12, \text { Max LET }=75.6\end{array}$ \\
\hline $66252-000$ & 707 & Optocoupler & Micropac & LBNL & $\begin{array}{l}\text { Vcc }=3.3 \mathrm{~V}, \\
\text { SET: }-3.3 \mathrm{~V}, 400 \mathrm{~ns} \\
\text { Weibull: } \mathrm{L}_{0}=9.5, \sigma_{0}=2.0 \mathrm{E}-4, \mathrm{~W}=14, \mathrm{~S}=1.9, \text { Max LET }=60\end{array}$ \\
\hline ILD2 & V937H & Optocoupler & Vishay & LNBL & $\begin{array}{l}\text { SET1: }-3 \mathrm{~V}, 40 \mathrm{us} \\
\text { Weibull: } \mathrm{L}_{0}=9.5, \sigma_{0}=1.6 \mathrm{E}-3, \mathrm{~W}=20, \mathrm{~S}=2 \\
\text { SET2: }-2 \mathrm{~V}, 200 \mathrm{~ns} \\
\text { Weibull: } \mathrm{L}_{0}=3, \sigma_{0}=4.0 \mathrm{E}-4, \mathrm{~W}=8, \mathrm{~S}=1.2, \mathrm{Max} \mathrm{LET}=60\end{array}$ \\
\hline HS-4423 & $* *$ & FET driver & Intersil & LNBL & No SET, Bias at 13V, Max LET=75.7 \\
\hline VRG8662 & 1002 & LDO reg & Aeroflex & TAMU & SET at high LET $(56>$ SET $>=87.1)$, No DSEE \\
\hline IS-1009RH & 451 & 2.5V Reference & Intersil & TAMU & $\begin{array}{l}\text { Vsupply }=13 \mathrm{~V}, \text { Vout }=2.5 \mathrm{~V} \\
\text { SET1: } 0.5,-2 \mathrm{~V}, 5 \mathrm{us} . \\
\text { Weibull: } \mathrm{L}_{0}=5.7, \sigma_{0}=8.1 \mathrm{E}-4, \mathrm{~W}=14.6, \mathrm{~S}=1 \\
\text { SET2: } \text { same as } 1 \text { but } 45 \mathrm{us} \text {. } \\
\text { Weibull: } \mathrm{L}_{0}=5, \sigma_{0}=7.4 \mathrm{E}-5, \mathrm{~W}=17.7, \mathrm{~S}=1.226 \text {, } \\
\text { No DSEE, Max LET }=87.1\end{array}$ \\
\hline 66266 & 1119 & Optocoupler & Micropac & TAMU & $\begin{array}{l}\text { SET: }-700 \mathrm{mV} \text { to } 1.1 \mathrm{~V} \text { (from Vout), } 50-70 \mathrm{us}, \\
\text { Weibull: } \mathrm{L}_{0}=1, \sigma_{0}=2.9 \mathrm{E}-3, \mathrm{~W}=21.4, \mathrm{~S}=5, \\
\text { Vout }=15 \mathrm{~V}, \text { Max LET }=60\end{array}$ \\
\hline IRHLG77214 & 1126 & $\begin{array}{l}\text { 250V Quad N-Channel } \\
\text { MOSFET } \\
\end{array}$ & IR & TAMU & No SEB at $182 \mathrm{~V}$ at $\mathrm{LET}=87.1$, gate leakage noted on 2 units \\
\hline IS-1009RH & 451 & Linear bipolar & Intersil & TAMU & $\begin{array}{l}\text { SET: } \\
\text { Pos: } 600 \mathrm{mV} / 1.5 \mathrm{us} \\
\text { Neg: }-1.75 \mathrm{~V} / 19 \mathrm{us} \text {, } \\
\text { No SEL, Max LET }=77.3\end{array}$ \\
\hline OLF-300 & 504 & Optocoupler & Isolink & TAMU & All transients below $3 \mathrm{~V}, \mathrm{Max}$ LET $=77.3$ \\
\hline GoPro Hero 3 & $* *$ & Camera & GoPro & & Camera survived with many SEE \\
\hline MKD25PA128IO-672A & $* *$ & Solid State Drive Assy & Memkor & IUCF & $\begin{array}{l}\text { DSEE: Unit failed to respond to power cycle. } \\
\text { Bendel } \mathrm{A}=13.08\end{array}$ \\
\hline IRHF7330SE & 1406 & N-Channel MOSFET & IR & TAMU & Degradation observed at 1E5, Max LET 77.3 \\
\hline IR2110L4 & 1146 & FET driver & IR & TAMU & $\begin{array}{l}\text { SET: State changes and transients, } \\
\text { No DSEE, Max LET }=77.3\end{array}$ \\
\hline HERO4 & & Camera & GoPro & IUCF & Numerous SEE, no DSEE \\
\hline IRHQ57214SE & 1436 & $\begin{array}{l}\text { 250V N-Channel } \\
\text { MOSFET }\end{array}$ & IR & TAMU & $\begin{array}{l}\text { Pass at } \mathrm{Vds}=171 \mathrm{~V}, \mathrm{LET}=77.3 . \mathrm{SET}=+/-4 \mathrm{~V} \text { on the drain, } \\
+/-1.75 \mathrm{~V} \text { on the source. }<0.5 \mathrm{us} \text { duration }\end{array}$ \\
\hline $\begin{array}{l}\text { DFI downselect: } \\
\text { NSW-5FT-TGE-2, } \\
\text { Ethos Lite, X52000001- } \\
01, \text { X92000001-01 }\end{array}$ & $* *$ & 5 Port Switch & TTC & IUCF & No DSEE after $1 \mathrm{E} 10 / \mathrm{cm}^{2}$ \\
\hline IRHF7110SCS & 1130 & $\begin{array}{l}\text { 100V, N-Channel } \\
\text { MOSFET }\end{array}$ & IR & TAMU & $\mathrm{Vds}=20 \mathrm{~V}$, no DSEE or degradation. \\
\hline
\end{tabular}




\begin{tabular}{|c|c|c|c|c|c|}
\hline IRHYS67234T3 & 1439 & $\begin{array}{l}\text { 250V, N-Channel } \\
\text { MOSFET }\end{array}$ & IR & TAMU & $\mathrm{Vds}=240 \mathrm{~V}$, No DSEE or degradation. \\
\hline $2 \mathrm{~N} 3439$ & 1148 & 350V, NPN BJT & Microsemi & TAMU & $\begin{array}{l}\text { SET }=1.8 \mathrm{~V} /<0.5 \mathrm{us}, \sigma_{0}=2.33 \mathrm{E}-3 \\
\mathrm{Vce}=166 \mathrm{~V}, \text { No DSEE }\end{array}$ \\
\hline OP27AL & $0936 \mathrm{~A}$ & Linear Bipolar & $\mathrm{AD}$ & LBNL & DSEE: Above LET $=45.6$ \\
\hline Hero3 & $* *$ & $\begin{array}{l}\text { Camera and associated } \\
\text { electronics }\end{array}$ & GoPro & NSRL & DSEE at LET $=4.2$ \\
\hline MACQ-500E-2 & ** & Overhead module & TTC & FBPTC & Unit failed in less than $1 \mathrm{E} 10 / \mathrm{cm} 2$ \\
\hline RH1013MW & $1320 \mathrm{~A}$ & Precision Op-amp & $\begin{array}{c}\text { Linear } \\
\text { Technology }\end{array}$ & LBNL & largest SET: $5.7 \mathrm{~V}, 13$ usec, No DSEE \\
\hline OP484 & $1009 \mathrm{~A}$ & Op-amp & $\mathrm{AD}$ & LBNL & $\begin{array}{l}\text { No SEDR. Trigger }>250 \mathrm{mV}, 1 \mathrm{~V} / 1 \mathrm{uS} \text { max transient, } \\
\text { Weibull: } \mathrm{L}_{0}=0.1, \sigma_{0}=2.8 \mathrm{E}-3, \mathrm{~W}=37, \mathrm{~S}=3, \mathrm{Vcc}=17.7 \mathrm{~V}\end{array}$ \\
\hline 900613 & ** & SSD & VisionTek & NSRL & DSEE: $\sigma_{0}=1.12 \mathrm{E}-6$ at LET $=>4.2$ (upper limit) \\
\hline KVR16LS11/4 & ** & DRAM & Kingston & NSRL & DSEE: $\sigma_{0}=4.5 \mathrm{E}-7$ at LET $=>4.2$ (upper limit) \\
\hline IRHF7110SCS & 1130 & $\begin{array}{l}\text { N-Channel 100V } \\
\text { MOSFET }\end{array}$ & $\begin{array}{l}\text { International } \\
\text { Rectifier }\end{array}$ & TAMU & $\begin{array}{l}\text { No DSEE or degradation, } \mathrm{Vds}=20 \mathrm{~V} \\
\text { Max LET }=75\end{array}$ \\
\hline 88E1111-NDC2 & 1307 & PHY & Marvell & TAMU & $\begin{array}{l}\text { SEU "Packet Errors" } \\
\text { Weibull: } \mathrm{L} 0=0.1, \sigma_{0}=1.3 \mathrm{E}-3, \mathrm{~W}=70, \mathrm{~S}=1.3 \\
\text { SEFI Weibull: } \\
\text { L0 }=0.1, \sigma_{0}=9.0 \mathrm{E}-6, \mathrm{~W}=70, \mathrm{~S}=0.7 \text {, cleared with RESET or power cycle } \\
\text { Max LET }=77\end{array}$ \\
\hline WIL6120 & $* *$ & Radio controller & Wilocity & NSRL & $\begin{array}{l}\text { SEE } \sigma_{0}=3.65 \mathrm{E}-4 \text { at LET }=>4.2 \text { (upper limit for self-recovering SEE) } \\
\text { SEE } \sigma_{0}=5.58 \mathrm{E}-4 \text { at } \mathrm{LET}=>4.2 \text { (upper limit for SEE requiring } \\
\text { intervention) }\end{array}$ \\
\hline NSW-12GT-1 & $* *$ & 12 bit Ethernet Switch & TTC & MGH & $\begin{array}{l}\text { SEFI requiring power cycle: Bendel } A=13.07 \\
\text { DSEE: Bendel } A=18.03\end{array}$ \\
\hline SN54AC14W & $1131 \mathrm{~A}$ & $\begin{array}{l}\text { Hex inverter/Schmitt } \\
\text { trigger }\end{array}$ & TI & LBNL & $\begin{array}{l}\text { SET }=+/-200 \mathrm{mV}, \text { No SET at } 48.2 \\
\text { Upper limit } \sigma_{0}=3.85 \mathrm{E}-6 \text { at LET }=75.6\end{array}$ \\
\hline $\begin{array}{l}\text { NSW-12GT-1 (power } \\
\text { supply only) }\end{array}$ & $* *$ & $28 \mathrm{~V}$ to $5 \mathrm{~V}$ converter & TTC & NSRL & DSEE $\sigma 0=1.00 \mathrm{E}-5$ at LET $=>14.2$ (upper limit) \\
\hline Hero4 Black & ** & $\begin{array}{l}\text { Portable HD Digital } \\
\text { Video Camera }\end{array}$ & GoPro & NSRL & DSEE $\sigma_{0}=1.36 \mathrm{E}-6$ at LET $=>14.2$ (upper limit) \\
\hline D54250WYK1 & ** & SBC & NUC Intel & NSRL & DSEE $\sigma_{0}=9.04 \mathrm{E}-5$ at LET $=>14.2$ (upper limit) \\
\hline MAGBES-21HS & $* *$ & 5 Port Ethernet Switch & $\begin{array}{l}\text { MPLAG } \\
\text { Elektronikuntern } \\
\text { ehmen }\end{array}$ & NSRL & DSEE $\sigma_{0}=1.87 \mathrm{E}-6$ at LET $=>14.2$ (upper limit) \\
\hline PIP37-1 & $* *$ & Ruggedized SBC & $\begin{array}{l}\text { MPLAG } \\
\text { Elektronikuntern } \\
\text { ehmen }\end{array}$ & NSRL & DSEE $\sigma_{0}=5.06 \mathrm{E}-4$ at LET $=>14.2$ (upper limit) \\
\hline $\begin{array}{l}\text { GoPro Htr 6/8/2015 } \\
\text { DSS }\end{array}$ & ** & Heater board & $\begin{array}{l}\text { Deep Space } \\
\text { Systems }\end{array}$ & NSRL & DSEE $\sigma_{0}=5.05 \mathrm{E}-7$ at LET $=>14.2$ (upper limit) \\
\hline NSW-8GT-TG-D-1 & $* *$ & 8 Port Network Switch & TTC & MGH & SEE: Bendel A=14.81 \\
\hline $175-0600-0103 \mathrm{~L}$ & $* *$ & 5 Port Ethernet Switch & Gadget Smyth & MGH & $\begin{array}{l}\text { SEE Bendel } A=12.73 \text { (self-recovering SEE) } \\
\text { SEE Bendel } A=14.33 \text { (SEE requiring intervention) } \\
\text { DSEE: Bendel } A=15.01\end{array}$ \\
\hline ATXMEGA128A1U & 1504 & Microcontroller & Atmel & TAMU & $\begin{array}{l}\text { Stuck bits }- \text { stuck at } 0 \text {. } \\
\text { Weibull: } \mathrm{L}_{0}=16.2, \sigma_{0}=7.9 \mathrm{E}-5, \mathrm{~W}=101.3, \mathrm{~S}=2.3\end{array}$ \\
\hline
\end{tabular}




\begin{tabular}{|c|c|c|c|c|c|}
\hline & & & & & tested powered to LET $=77$ \\
\hline 66183 & $* *$ & OptoCoupler & Microsemi & NSRL & No SEE noted, using VDBP method \\
\hline JANSF2N7484T3 & $* *$ & MOSFET & IR & NSRL & No SEE noted, using VDBP method \\
\hline QT625LBM-25.8 MHZ & $* *$ & Oscillator & Q-Tech & NSRL & $\begin{array}{l}\text { SEFI: Weibull: } \mathrm{L}_{0}=1.0, \sigma_{0}=7.9 \mathrm{E}-6, \mathrm{~W}=5.9, \mathrm{~S}=5.01 \\
\text { Max LET }=60+\text { using VDBP method }\end{array}$ \\
\hline IS9-139ASRH & $* *$ & Voltage Comparitor & Intersil & NSRL & No DSEE to LET $=60+$ \\
\hline IS9-1825ASRH & $* *$ & PWM Controller & Intersil & NSRL & No DSEE to LET $=60+$ \\
\hline SPT6235M-NPN & $* *$ & Transistor & SSDI & NSRL & No SEE noted to LET $=60+$ using VDBP method \\
\hline LMC6482A-HCI & $* *$ & Op Amp & $\begin{array}{c}\text { Texas } \\
\text { Instrument }\end{array}$ & TAMU & SETs application dependent \\
\hline 4011BEDIE2HR & $* *$ & NAND Gate & ST-Micro & LBNL & Worst case SET: $-370 \mathrm{mV}, 20$ nsecs, Max LET $=77.5$ \\
\hline $74 \mathrm{HC} 02$ & 1145 & $\begin{array}{l}\text { Quad 2-inout NOR } \\
\text { Gate }\end{array}$ & Fairchild Semi & TAMU & No SETS, No DSEE \\
\hline RIC7113A4SCS & 1424 & $\begin{array}{l}\text { High/Low side Gate } \\
\text { Driver } \\
\end{array}$ & IR & TAMU & Longest Dropout 550 msecs, No DSEE, Max LET=77 \\
\hline 74AC00 & 1507 & $\begin{array}{l}\text { Quad 2-inout NAND } \\
\text { Gate }\end{array}$ & Fairchild Semi & LBNL & Worst SET $=+/-300 \mathrm{mV}, 58$ nsecs, No DSEE, Max LET=75 \\
\hline HCPL-523K \#300 & 1314 & Dual Logic Opto & $\begin{array}{c}\text { Avago } \\
\text { Technologies }\end{array}$ & LBNL & No DSEE, Max LET=75 \\
\hline OLH249 & 1548 & Opto - Hybrid & Isolink & LBNL & Worst case SET: $1.28 \mathrm{~V}, 70$ usecs, Max LET $=75$ \\
\hline RH6105 & $* *$ & Current Sense Amp & Linear Tech & LBNL & Worst case SET: $3 \mathrm{~V} /-1 \mathrm{~V},<9$ usecs, Max LET $=75$ \\
\hline XCF128XFTG64C & $\begin{array}{l}\text { 99A3R } \\
\text { VS, MYS } \\
99538\end{array}$ & $\begin{array}{l}\text { FLASH non-volatile } \\
\text { Memory }\end{array}$ & Xilin $x$ & NSRL & 1 bit-flip was observed at LET $\sim 40$, VDBP method \\
\hline SN54AHC244W & 726 & Octal Buffer & TI & TAMU & High Temp No DSEE, Max LET=77 \\
\hline IS42S16400J-5BL & $* *$ & SDRAM & ISSI & NSRL & $\begin{array}{l}\text { Bitflip Error: Weibull: } \mathrm{L}_{0}=.01, \sigma_{0}=4.6 \mathrm{E}-1, \mathrm{~W}=110, \mathrm{~S}=2.9 \\
\text { No DSEE: } 7.68 \mathrm{E} 4 / \mathrm{cm}^{2} \text { at LET }=39 \text {. }\end{array}$ \\
\hline SI7415DN-T1-GE3 & $* *$ & P Channel MOSFET & Vishay & NSRL & No DSEE at LET $=29,12 \mathrm{~V}$ \\
\hline DS2411R+T\&R & $* *$ & SCSI terminator & Maxim & NSRL & No DSEE for $7.79 \mathrm{E} 4 / \mathrm{cm}^{2}$ at $\mathrm{LET}=39$ \\
\hline TMP006AIYZFR & $* *$ & Thermopile & TI & NSRL & $\begin{array}{l}\text { Local temp error: Weibull: } \mathrm{L}_{0}=10, \sigma_{0}=2.5 \mathrm{E}-5, \mathrm{~W}=14, \mathrm{~S}=4 \text {. } \\
\text { Obj temp error: Weibull: } \mathrm{L}_{0}=5, \sigma_{0}=5 \mathrm{E}-0, \mathrm{~W}=0.1, \mathrm{~S}=10 \text {. } \\
\text { No DSEE: } 7.37 \mathrm{E} 4 / \mathrm{cm}^{2} \text { at } \mathrm{LET}=29 \text {. }\end{array}$ \\
\hline 74AUP1G157GW & $* *$ & Analog Mux & NXP & NSRL & No SEE at $1.61 \mathrm{E} 5 / \mathrm{cm}^{2}$ at $\mathrm{LET}=39$ \\
\hline $\begin{array}{l}\text { ASDMPC-10.000MHZ- } \\
\text { RT-T } \\
\end{array}$ & $* *$ & Oscillator & Abracon & NSRL & Failed immediately. \\
\hline DS1339A & $* *$ & Clock & $\begin{array}{c}\text { Maxim } \\
\text { Integrated }\end{array}$ & NSRL & $\begin{array}{l}\text { Soft Errors: Weibull: } \mathrm{L}_{0}=12, \sigma_{0}=9 \mathrm{E}-5, \mathrm{~W}=0.1, \mathrm{~S}=10 \\
\text { No DSEE: } 7.54 \mathrm{E} 4 / \mathrm{cm}^{2} \text { at LET }=29\end{array}$ \\
\hline SN74LVC1G66DCKR & $* *$ & Analog switch & TI & NSRL & No SEE, DSEE: $7.62 E 4 / \mathrm{cm} 2$ at LET=29 \\
\hline N25Q128 & $* *$ & Flash memory & Micron & NSRL & $\begin{array}{l}\text { Read Error: Weibull: } \mathrm{L}_{0}=1, \sigma_{0}=4 \mathrm{E}-5, \mathrm{~W}=27, \mathrm{~S}=2 \\
\text { No DSEE: } 7.5 \mathrm{E} 4 / \mathrm{cm}^{2} \text { at LET }=29\end{array}$ \\
\hline $\begin{array}{l}\text { SN74CBTLV3257RSV } \\
\text { R }\end{array}$ & $* *$ & $\begin{array}{l}\text { Mux/demux and Bus } \\
\text { switch }\end{array}$ & TI & NSRL & $\begin{array}{l}\text { No SEE noted at } 7.55 \mathrm{E} 4 / \mathrm{cm}^{2} \\
\text { DSEE: } 7.55 \mathrm{E} 4 / \mathrm{cm}^{2} \text { at } \mathrm{LET}=29\end{array}$ \\
\hline NC7SZ125M5X & $* *$ & Tri-state buffer & $\begin{array}{c}\text { On } \\
\text { Semiconductor }\end{array}$ & NSRL & No SEE noted. DSEE: $7.55 E 4 / \mathrm{cm}^{2}$ at LET $=29$. \\
\hline SN74AHC1G08DBVR & $* *$ & Quad AND Gate & TI & NSRL & No SEE noted. DSEE: $7.55 E 4 / \mathrm{cm}^{2}$ at LET $=29$ \\
\hline $\begin{array}{l}\text { SN74LVC1G125BDV } \\
\text { R }\end{array}$ & $* *$ & Tri-state buffer & & NSRL & No SEE or DSEE: $7.55 E 4 / \mathrm{cm}^{2}$ at LET=29 \\
\hline
\end{tabular}




\begin{tabular}{|c|c|c|c|c|c|}
\hline CB3LV-3C-25MHZ & $* *$ & Oscillator & & NSRL & $\begin{array}{l}\text { No SEE at } 7.55 \mathrm{E} 4 / \mathrm{cm}^{2} \\
\text { DSEE: } 7.55 \mathrm{E} 4 / \mathrm{cm} 2 \text { at LET }=39\end{array}$ \\
\hline FDMC86139 & $* *$ & $\begin{array}{c}\text { P-Channel MOSFET, } \\
100 \mathrm{~V}\end{array}$ & $\begin{array}{l}\text { Fairchild/ON } \\
\text { semi }\end{array}$ & NSRL & No DSEE for $1 \mathrm{E} 5 / \mathrm{cm}^{2}$ at LET $=11.75$ \\
\hline TLV70133 & $* *$ & Linear Regulator & TI & NSRL & No DSEE for $1 \mathrm{E} 5 / \mathrm{cm}^{2}$ at LET $=11.75$ \\
\hline TPS22929 & *** & Single Load Switch & TI & NSRL & No DSEE for $1 \mathrm{E} 5 / \mathrm{cm}^{2}$ at LET $=15$ \\
\hline CB3LV & $* *$ & Oscillator & $\begin{array}{l}\text { CTS-Frequency } \\
\text { controls }\end{array}$ & NSRL & No DSEE for $1 \mathrm{E} 5 / \mathrm{cm}^{2}$ at $\mathrm{LET}=15$ \\
\hline TPS73601 & $* *$ & $\begin{array}{c}\text { Linear Voltage } \\
\text { Regulator }\end{array}$ & TI & NSRL & No DSEE for $1 \mathrm{E} 5 / \mathrm{cm}^{2}$ at LET $=11.75$ \\
\hline & $* *$ & & & & \\
\hline NAND Flash & *** & & & NSRL & No DSEE for $1 \mathrm{E} 5 / \mathrm{cm}^{2}$ at LET $=11.75$ \\
\hline DP83640 & ** & $\begin{array}{c}\text { Precision } \\
\text { PHYTERTM IEEE } \\
\text { 1588 Transceiver }\end{array}$ & TI & NSRL & No DSEE for $1 \mathrm{E} 5 / \mathrm{cm}^{2}$ at $\mathrm{LET}=11.75$ \\
\hline OPA2209 & ** & Precision Op Amps & TI & NSRL & No DSEE for $1 \mathrm{E} 5 / \mathrm{cm}^{2}$ at LET $=11.75$ \\
\hline LM4040 & $* *$ & Voltage Reference & ON-Semi & NSRL & No DSEE for $1 \mathrm{E} 5 / \mathrm{cm}^{2}$ at LET $=11.75$ \\
\hline LT3092 & $* *$ & $\begin{array}{c}\text { 200mA 2-Terminal } \\
\text { Programmable Current } \\
\text { Source }\end{array}$ & $\begin{array}{c}\text { Linear } \\
\text { Technology }\end{array}$ & NSRL & No DSEE for $1 \mathrm{E} 5 / \mathrm{cm}^{2}$ at $\mathrm{LET}=11.75$ \\
\hline OMAP L138 & ** & ARM/DSP Processor & & NSRL & $\begin{array}{l}\text { L2 Error: Weibull: } \mathrm{L}_{0}=1, \sigma_{0}=2.23 \mathrm{E}-2, \mathrm{~W}=57, \mathrm{~S}=1.04 \\
\text { Overcurrent: } \mathrm{L}_{0}=24, \sigma_{0}=3 \mathrm{E}-4 \\
\text { L3 Error: Weibull: } \mathrm{L}_{0}=1, \sigma_{0}=2.88 \mathrm{E}-4, \mathrm{~W}=16.6, \mathrm{~S}=1.166 \\
\text { L1D Error: Weibull: } \mathrm{L}_{0}=1, \sigma_{0}=8.57 \mathrm{E}-3, \mathrm{~W}=120.8, \mathrm{~S}=1.2 \\
\text { CPU Functional Interrupt: } \\
\text { Weibull: } \mathrm{L}_{0}=1, \sigma_{0}=4.15 \mathrm{E}-4, \mathrm{~W}=55.2, \mathrm{~S}=1.2 \\
\text { PRU Functional Interrupt: } \\
\text { Weibull: } \mathrm{L}_{0}=3, \sigma_{0}=2 \mathrm{E}-4, \mathrm{~W}=0.1, \mathrm{~S}=10 \\
\text { Software error: } \\
\text { Weibull: } \mathrm{L}_{0}=3, \sigma_{0}=2.1 \mathrm{E}-4, \mathrm{~W}=19.5, \mathrm{~S}=1.628\end{array}$ \\
\hline FRO15L3EZ & ** & $\begin{array}{l}\text { Reverse Polarity } \\
\text { Device }\end{array}$ & $\begin{array}{c}\text { ON } \\
\text { Semiconductor }\end{array}$ & NSRL & No DSEE: LET=39, $1 \mathrm{E} 6 / \mathrm{cm}^{2}$ \\
\hline TPS62142 & $* *$ & Temperature Sensor & TI & NSRL & 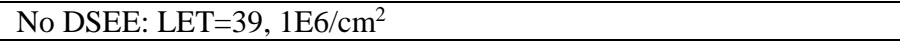 \\
\hline TPS73601 & $* *$ & $\begin{array}{l}\text { Remote Power } \\
\text { Regulator }\end{array}$ & TI & NSRL & No DSEE: $\mathrm{LET}=39,1 \mathrm{E} 6 / \mathrm{cm}^{2}$ \\
\hline RM48L950 & ** & $\begin{array}{l}\text { 16/32 BIT RISC Flash } \\
\text { Microcontroller }\end{array}$ & TI & NSRL & $\begin{array}{l}\text { RAM single bit error: Weibull: } \mathrm{L}_{0}=3, \sigma_{0}=1.44 \mathrm{E}-2, \mathrm{~W}=19.5, \mathrm{~S}=1.628 \\
\text { Software error: Weibull: } \mathrm{L}_{0}=1, \sigma_{0}=2.1 \mathrm{E}-4, \mathrm{~W}=27.3, \mathrm{~S}=0.869 \\
\text { Flash error: Weibull: } \mathrm{L}_{0}=2.7, \sigma_{0}=5.5 \mathrm{E}-4, \\
\text { SEFI: Weibull: } \mathrm{L}_{0}=1, \sigma_{0}=6.0 \mathrm{E}-4 \\
\text { No DSEE: } \mathrm{LET}=39,9 \mathrm{E} 3 / \mathrm{cm}^{2}\end{array}$ \\
\hline KSZ8895 & $* *$ & Ethernet Switch & Microchip Tech. & NSRL & DSEE: Weibull: $\mathrm{L} 0=12, \mathrm{v}=1.59 \mathrm{E}-3, \mathrm{~W}=5.4, \mathrm{~S}=2.376$ \\
\hline MT29F32G08 & ** & NAND flash & Micron & NSRL & $\begin{array}{l}\text { MTD2 Byte error : } \\
\text { Weibull: } \mathrm{L}_{0}=3, \sigma_{0}=1.3 \mathrm{E}-1, \mathrm{~W}=66.15, \mathrm{~S}=2.38 . \mathrm{ECC} \text { was very effective } \\
\text { at lower } \mathrm{LET} \\
\text { Erase Failed Error: } \\
\text { Weibull: } \mathrm{L}_{0}=4, \sigma_{0}=1.3 \mathrm{E}-4, \mathrm{~W}=23.8, \mathrm{~S}=0.462 \\
\text { MTD2 Bad Block Error: Weibull } \mathrm{L}_{0}=2, \sigma_{0}=1.0 \mathrm{E}-4\end{array}$ \\
\hline
\end{tabular}




\begin{tabular}{|c|c|c|c|c|c|}
\hline & & & & & No DSEE: LET=51, 5E4/cm² \\
\hline NVH0505 & $* *$ & DC/DC converter & & NSRL & No DSEE to LET $=51,9 \mathrm{E} 3 / \mathrm{cm} 2$ \\
\hline AD5622 & $* *$ & Bias converter DAC & Analog Devices & NSRL & No SEE or DSEE at LET $=24,1 \mathrm{E} 4 / \mathrm{cm}^{2}$ \\
\hline AD7991 & $* *$ & Bias converter ADC & Analog Devices & NSRL & No SEE or DSEE at LET $=24,1 \mathrm{E} 4 / \mathrm{cm}^{2}$ \\
\hline MAX9619 & $* *$ & Remote Temp sensor & Maxim & NSRL & No SEE or DSEE at LET $=24$ \\
\hline AGLN250V2-VQG100 & $* *$ & Flash FPGA & Microsemi & NSRL & $\begin{array}{l}\text { SEFI: } \mathrm{L}_{0}=18, \mathrm{~S} 0=3 \mathrm{E}-5 \\
\text { No DSEE to } \mathrm{LET}=51,9 \mathrm{E} 3 / \mathrm{cm}^{2}\end{array}$ \\
\hline MT46H64M16LFB & $* *$ & DDR memory & Micron & NSRL & $\begin{array}{l}\text { Single bit error: } \\
\text { Weibull: } \mathrm{L}_{0}=9, \sigma_{0}=9 \mathrm{E}-3, \mathrm{~W}=10, \mathrm{~S}=1.274 \\
\text { Multiple bit error: } \\
\text { Weibull: } \mathrm{L}_{0}=9, \sigma_{0}=1 \mathrm{E}-4\end{array}$ \\
\hline LAN8710A-EZC & $* *$ & Ethernet PHY & Microchip Tech & NSRL & $\begin{array}{l}\text { SEFI: } \mathrm{L}_{0}=2, \sigma_{0}=1 \mathrm{E}-4 \\
\text { No DSEE to } \mathrm{LET}=39,1 \mathrm{E} 4 / \mathrm{cm}^{2}\end{array}$ \\
\hline 74LCX573 & $* *$ & Octal Latch & $\begin{array}{c}\text { Fairchild } \\
\text { Semiconductor }\end{array}$ & NSRL & No SEE or DSEE at LET $=24,1 E 4 / \mathrm{cm}^{2}$ \\
\hline DS90LV047/48 & $* *$ & $\begin{array}{c}\text { Quad Line } \\
\text { Driver/receiver }\end{array}$ & TI & NSRL & No SEE or DSEE to LET $=39$ \\
\hline TIMEPIX & $* *$ & Radiation sensor & Timepix & NSRL & No SEE or DSEE to LET $=39$ \\
\hline MAX6692 & $* *$ & Temp Sensor & Maxim & NSRL & No DSEE to LET $=26.5$ \\
\hline LTM4644IY & $* *$ & Quad switcher & & NSRL & DSEE around LET $=3$ \\
\hline TPS54295RSAT & $* *$ & Switching Regulator & TI & NSRL & $\begin{array}{l}\text { SEFI: Weibull: } \mathrm{L}_{0}=4, \sigma_{0}=4.5 \mathrm{E}-5, \mathrm{~W}=3, \mathrm{~S}=2 \\
\text { DSEE at LET }=19\end{array}$ \\
\hline LTC3646 & $* *$ & Step down regulator & $\begin{array}{c}\text { Linear } \\
\text { Technology }\end{array}$ & NSRL & $\begin{array}{l}\text { Trigger: Weibull: } \mathrm{L}_{0}=3, \sigma_{0}=2.65 \mathrm{E}-4, \mathrm{~W}=44, \mathrm{~S}=1.38 \\
\text { SEFI: Weibull: } \mathrm{L}_{0}=39, \sigma_{0}=9 \mathrm{E}-5 \\
\text { No DSEE to LET }=39,9 \mathrm{E} 4 / \mathrm{cm}^{2}\end{array}$ \\
\hline LM3880Q & $* *$ & Power Sequencer & $\mathrm{TI}$ & NSRL & $\begin{array}{l}\text { No SEE to LET }=24,9 E 3 / \mathrm{cm}^{2} \\
\text { No DSEE to LET }=39\end{array}$ \\
\hline INA230 & $* *$ & $\begin{array}{l}\text { Bidirectional } \\
\text { current/power monitor }\end{array}$ & TI & NSRL & $\begin{array}{l}\text { Trigger error: Weibull: } \mathrm{L}_{0}=24, \sigma_{0}=7 \mathrm{E}-5, \mathrm{~W}=35, \mathrm{~S}=1 \\
\text { Register error: Weibull: } \mathrm{L}_{0}=24, \sigma_{0}=6 \mathrm{E}-5, \mathrm{~W}=15, \mathrm{~S}=1\end{array}$ \\
\hline $\begin{array}{l}\text { OMH3040 } \\
\text { OMH3005S }\end{array}$ & 1523 & Hall-effect Sensor & $\begin{array}{l}\text { TT Electronics / } \\
\text { Optek }\end{array}$ & LBNL & $\begin{array}{l}\text { Worst case SET: } 15 \mathrm{~V} \text { to ground, } 4 \text { usecs, } \\
\text { No DSEE at LET }=77.5\end{array}$ \\
\hline 4011BEDIE2HR & $* *$ & NAND Gate & ST-Micro & LBNL & $\begin{array}{l}\text { Worst case SET: }-370 \mathrm{mV}, 20 \mathrm{nsecs} \text {, } \\
\text { No DSEE at LET }=77\end{array}$ \\
\hline 74HC02 & 1145 & $\begin{array}{l}\text { Quad 2-inout NOR } \\
\text { Gate }\end{array}$ & Fairchild Semi & TAMU & No SET or DSEE at LET $=77$ \\
\hline RIC7113A4SCS & 1424 & $\begin{array}{l}\text { High/Low side Gate } \\
\text { Driver }\end{array}$ & IR & TAMU & Longest Dropout 550 msecs, No DSEE at 77 \\
\hline JANTXV2N3439UA & 1447 & High V Transistor & Microsemi & TAMU & $\begin{array}{l}\text { Worst case SET: } 2 \mathrm{~V} \text { and }-2.4 \mathrm{~V},<100 \text { nsecs, } \\
\text { No DSEE at LET }=77\end{array}$ \\
\hline ОМН3075 & _0043 & Hall-effect Sensor & $\begin{array}{l}\text { TT Electronics / } \\
\text { Optek }\end{array}$ & TAMU & $\begin{array}{l}\text { Worst case SET: } 15 \mathrm{~V} \text { to ground, } 5 \text { usecs, } \\
\text { No DSEE at LET }=75\end{array}$ \\
\hline
\end{tabular}


useful when assessing current parts against previously tested parts.

\section{TEST RESUlts AND DISCUSSION}

In this section, details for individual tests are discussed to provide more information as required. Additionally, test results analyses are discussed. The usage of the 1-parameter Bendel curve is discussed along with the development of the Weibull parameters from the high energy heavy ion test. Specific details of the VDBP test results analyses are also discussed.

\section{CONCLUSIONS}

We have presented proton test data and/or heavy ion test data results for a variety of piece parts and/or COTS units being considered for applications in a LEO or deep space radiation environment. Additionally, test data from high energy heavy ion testing (VDBP) has been discussed and presented. As NASA continues to develop plans for deep space missions, new radiation-related challenges will exist with the increased use of COTS parts and hardware. With limited budgets, designers are increasingly looking to published data in compendiums such as this to help make decisions on parts.

\section{ACKNOWLEDGMENT}

We would like to thank the support staff at LBNL, TAMU, Francis Burr and other proton facilities for the professional and excellent support. Additionally, we are very thankful to Mike Sivertz and Adam Rusek of NSRL for the continued expertise in helping make Variable Depth Bragg Peak testing and screening a reality.

\section{REFERENCES}

[1] C. K. Kouba, K. V. Nguyen, P. M. O’Neill, and C. R. Bailey, "Proton Radiation Test Results on COTS-Based Electronic Devices for NASAJohnson Space Center Spaceflight Projects", 2006 IEEE Radiation Effects Data Workshop", Ponte Vedra Beach, Fl, 2006, pp. 26-36.

[2] K. K. Allums, P. M. O'Neill, B. D. Reddell, C. R. Bailey, K. V. Nguyen, "Radiation Test Results on COTS and Non-COTS Electronic Devices for NASA Johnson Space Center Spaceflight Projects", 2012 IEEE Radiation Effects Data Workshop", Tucson, Az, 2012, pp. 1-9.

[3] S. Buchner, N. Kanyogoro, D. McMorrow, C.C. Foster, P. M. O'Neill and K. V. Nguyen, "Variable Depth Bragg Peak Method for Single Event Effects Testing" IEEE Trans. on Nucl. Sci., vol. 58, no. 6, pp. 2976-2982, Dec. 2011.

[4] N. J. H. Roche et al., "Validation of the Variable Depth Bragg Peak Method for Single Event Latchup Testing Using Ion Beam Characterization" IEEE Trans. on Nucl. Sci., vol. 61, no. 6, pp. 30613067, Dec. 2014.

[5] C. C. Foster, "Certification of Parts for Space With the Variable Depth Bragg Peak Method" IEEE Trans. on Nucl. Sci., vol. 59, no. 6, pp. 29092913, Dec. 2012.

[6] P. M. O'Neill, G. D. Badhwar and W. X. Culpepper, "Risk assessment for heavy ions of parts tested with protons" IEEE Trans. on Nucl. Sci., vol. 44, no. 6, pp. 2311-2314, Dec. 1997.

[7] P. M. O’Neill, G. D. Badhwar and W. X. Culpepper, "Internuclear cascade-evaporation model for LET spectra of $200 \mathrm{MeV}$ protons used for parts testing" IEEE Trans. on Nucl. Sci., vol. 45, no. 6, pp. 24672474, Dec. 1998

[8] C. C. Foster, P. M. O’Neill and C. K. Kouba, "Risk Assessment Based on Upset Rates From High Energy Proton Tests and Monte Carlo Simulations" IEEE Trans. on Nucl. Sci., vol. 55, no. 6, pp. 2962-2969, Dec. 2008.

[9] L. Hoffmann and V. Lam, "Qualification of an Automotive-Grade, Stateof-the-Art ASIC for Natural Space Applications" 2013 IEEE Radiation Effects Data Workshop", San Francisco, Ca., 2013, pp. 1-8.

[10] C. La Tessa, M. Sivertz, I. Chiang, D. Lowenstein and A. Rusek, "Overview of the NASA space radiation laboratory" Life Sciences in Space Research, vol. 11, pp. 18-23, Oct. 2016 